\title{
Luxación traumática aguda de rodilla. Indicaciones del uso del fijador externo en la urgencia. Revisión bibliográfica sistematizada
}

Acute knee dislocation. Indications for the use of the external fixator at the emergency room Deslocamento agudo traumático do joelho. Indicações para o uso do fixador externo na emergência

\section{Mateo Pamparato¹, Juan M. Del Castillo², Luis Francescoli}

\section{Resumen}

Introducción: la luxación traumática de la rodilla constituye una lesión rara dentro de la patología ortopédica. En muchas ocasiones su diagnóstico pasa desapercibido dado que se dan en el contexto de pacientes politraumatizados, y la falla en el mismo puede derivar en un pronóstico potencialmente letal para el compromiso vital del miembro lesionado.

Objetivo: realizar una revisión de la literatura disponible en los últimos veinte años acerca de cuáles son las indicaciones del uso del fijador externo (FFEE) en la luxación traumática aguda de rodilla en la urgencia.

Materiales y métodos: se realizó una búsqueda sistematizada a través de los buscadores electrónicos Cochrane, Lilacs, Scielo, Pubmed, Science direct y el portal Timbó. La misma alcanzó un total de 6.495 artículos, y de acuerdo con los criterios de inclusión y exclusión se seleccionaron 14 trabajos para realizarla.

Resultados: se destaca que la mayoría de los artículos encontrados son de nivel de evidencia IV. La utilización del uso de la fijación externa en la urgencia como parte de la estabilización temporal y el manejo inicial estaría indicado en los siguientes escenarios clínicos: pacientes politraumatizados, lesión vascular, luxación expuesta, inestabilidad de la articulación, luxación recidivante, luxofracturas, lesiones a nivel del aparato extensor, obesidad mórbida, intolerancia del uso de la férula u ortesis.

Conclusión: la sistematización y protocolización a la hora de la toma de decisiones permite disminuir de forma mayoritaria las complicaciones vinculadas a la patología traumática; éstas deben estar dirigidas a la estabilización del paciente en primera instancia, y en segunda instancia a estabilizar la articulación de la rodilla. La fijación externa tiene indicaciones en escenarios clínicos puntuales; sin embargo algunas de estas indicaciones aún son objeto de debate.

Palabras clave: Luxación aguda de rodilla

Fijadores externos

Ligamentos articulares.

Key words: $\quad$ Acute knee dislocation

External fixators

Ligaments, articular

\footnotetext{
1. Residente. Clínica de Traumatologia y Ortopedia. Facultad de Medicina,UDELAR.

2. Asistente. Clínica de Traumatologia y Ortopedia. Facultad de Medicina,UDELAR.

3. Profesor . Clínica de Traumatologia y Ortopedia. Facultad de Medicina,UDELAR.

Clínica de Traumatologia y Ortopedia. Facultad de Medicina,UDELAR.

Los autores declaran no tener conflictos de intereses.

Correspondencia: Dr. Mateo Pamparato. Correo electrónico: mateopamparato4@gmail.com.

Recibido: 23/2/2021

Aprobado: 16/6/2021

Attribution-NonCommercial 4.0 International (CC BY-NC 4.0)
} 


\section{Introducción}

La luxación traumática de la rodilla es la disrupción completa de la integridad de la articulación tibio femoral. Se la define comúnmente como un desgarro de al menos dos de las cuatro estructuras principales de la articulación: el ligamento cruzado anterior (LCA), el ligamento cruzado posterior (LCP), la esquina posteromedial (PMC) y la esquina posterolateral (PLC). Los términos luxación y lesión multiligamentaria a menudo se usan indistintamente ${ }^{(1-3)}$. Las luxaciones a menudo resultan en lesiones ligamentarias múltiples, pero no toda lesión ligamentaria múltiple debe ser interpretada como una luxación.

Es una lesión poco frecuente, que históricamente ha tenido un pronóstico variable y puede llevar, potencialmente, al compromiso vital del miembro lesionado. Representa entre $0,02 \%-0,2 \%$ de las lesiones ortopédicas y se ha demostrado, pese a que su manejo es controvertido, que el diagnóstico temprano tiene un impacto significativo en el resultado funcional a largo plazo ${ }^{(1-3)}$.

La primera publicación realizada sobre la luxación traumática de la rodilla data de 1825 , y fue realizada por Sir Astley Cooper en su tratado de fracturas y dislocaciones sobre las articulaciones. Declaró: "De ésta solo he visto un caso y, por lo tanto, concluyo que es una ocurrencia rara; y apenas hay accidentes a los que sea responsable el cuerpo que exijan más imperiosamente la amputación inmediata que estos" ${ }^{(3-7)}$. En 1850 Birkett realizó la descripción del primer intento de reducción de una luxación expuesta utilizando cloroformo como anestesia. Desafortunadamente, esta luxación de rodilla irreductible fue finalmente amputada ${ }^{(6,7)}$.

Existen diversos métodos de estabilización temporal de la rodilla en la fase inicial de la patología ${ }^{(8,11-23)}$. Sin embargo, las preferencias de los especialistas sobre las estrategias de inmovilización antes y después de la reconstrucción ligamentaria siguen siendo controvertidas $^{(1-3,9,10)}$

La interrogante que nos planteamos para este trabajo surge a raíz de que se ha hecho costumbre en nuestro medio utilizar siempre el FFEE en esta lesión independientemente del escenario clínico, por lo cual nos propusimos realizar una revisión de la literatura para determinar cuáles son las indicaciones puntuales del uso del FFEE como método de estabilización primario y temporal en la urgencia.

\section{Objetivo general}

Revisar la evidencia actual acerca de las indicaciones del uso del fijador externo en la urgencia en las primeras 72 horas en la luxación traumática aguda de la rodilla.

\section{Materiales y métodos}

Este trabajo es una revisión sistemática. La búsqueda bibliográfica se realizó durante el mes de agosto de 2020. Las fuentes documentales utilizadas fueron Cochrane, Lilacs, Scielo, Pubmed, ScienceDirect y el portal Timbó. Con respecto al intervalo de tiempo para la obtención de los documentos se incluyeron artículos publicados desde el 2000 hasta la fecha. Las palabras clave utilizadas fueron en inglés e incluyeron los términos Mesh: "Knee dislocation", "External fixation", "Multiple ligament knee injuries". Los términos booleanos incluidos fueron "AND" y "OR". Se buscaron además artículos de libre acceso, en castellano y en inglés, en pacientes mayores de dieciocho años y que no incluyeran estudios en animales.

\section{Criterios de inclusión}

Se seleccionaron aquellos trabajos donde se realizaba una descripción del manejo inicial de la patología en la urgencia, en idioma inglés y español, en pacientes con esqueleto maduro, en humanos, en politraumatizados, luxofracturas, lesiones expuestas, lesiones del aparato extensor, lesión vascular asociada, que mencionaran además el uso de fijadores externos y publicaciones que cumplieran con el criterio temporal establecido para la búsqueda (2000-2020).

\section{Criterios de exclusión}

Se excluyeron meta análisis, revisiones sistemáticas, reportes de casos, artículos que no incluyeran la valoración inicial y estudios que valoraran estrictamente a la reconstrucción ligamentaria o la función de la rodilla. Además, no se incluyeron trabajos que mencionaran: estudios en animales, esqueleto inmaduro, luxaciones traumáticas crónicas, luxaciones congénitas, luxaciones con lesiones ligamentarias previas, luxaciones en pacientes con prótesis de rodilla.

\section{Estrategia de búsqueda}

Para la selección bibliográfica se utilizó como guía para confeccionar el diagrama de flujo el protocolo de revisiones PRISMA (figura 1).

De la búsqueda inicial en las diferentes plataformas surgieron un total de 6.495 artículos. Realizada la búsqueda por dos revisores independientes, se llegó al mismo número de artículos. Colocando los filtros mencionados (últimos 20 años, humanos, artículos en inglés e español) se obtuvieron 2.077 artículos.

Luego de aplicar los criterios de exclusión, se obtuvieron 550 trabajos. Posteriormente se realizó una lectura rápida de los títulos de los trabajos, quedando así 90 artículos. Se procedió a leer los abstracts de cada uno de ellos y se descartaron 50, quedando así 40 trabajos para 
Tabla 1. Artículos incluidos en la revisión.

\begin{tabular}{|c|c|c|c|c|}
\hline Autor principal & Tipo de estudio & $\begin{array}{c}\text { Año de publicación/motor de } \\
\text { búsqueda }\end{array}$ & Nivel de evidencia & $N$ muestral \\
\hline Gigante, Facundo et al & Retrospectivo & 2016; Lilacs & IV & 9 \\
\hline De los Ríos Giraldo, Adolfo & Retrospectivo & 2005; Lilacs & IV & 16 \\
\hline Carredano, Xabier et al & Restrospectivo & 2017; Lilacs & IV & 11 \\
\hline Kupczik, Fabiano et al & Observacional descriptivo & 2012; Lilacs & IV & 20 \\
\hline Stannard, James & Retrospectivo & 2016; Pubmed & IV & 27 \\
\hline Scarcella, Nicholas R et al & Retrospectivo & 2017; Pubmed & IV & 119 \\
\hline Levy, Bruce R et al & Prospectivo & 2010; Pubmed & IV & 9 \\
\hline Zamir, Mehroze et al & Prospectivo & 2014; Pubmed & IV & 9 \\
\hline Moatshe, Gilbert et al & Prospectivo & 2017; Pubmed & III & 303 \\
\hline López Hualda, A. et al & Retrospectivo & 2011; ScienDirect & IV & 10 \\
\hline Lustig, S et al & Prospectivo, Multicéntrico & 2009; ScienceDirect & IV & 67 \\
\hline Chang- Cheng, Liu et al & Retrospectivo & 2016; ScienceDirect & IV & 15 \\
\hline Novoa Sierra, B et al & Retrospectivo & 2015; ScienceDirect & IV & 11 \\
\hline Teissier, Victoria et al & Retrospectivo & 2019; ScienceDirect & IV & 16 \\
\hline
\end{tabular}

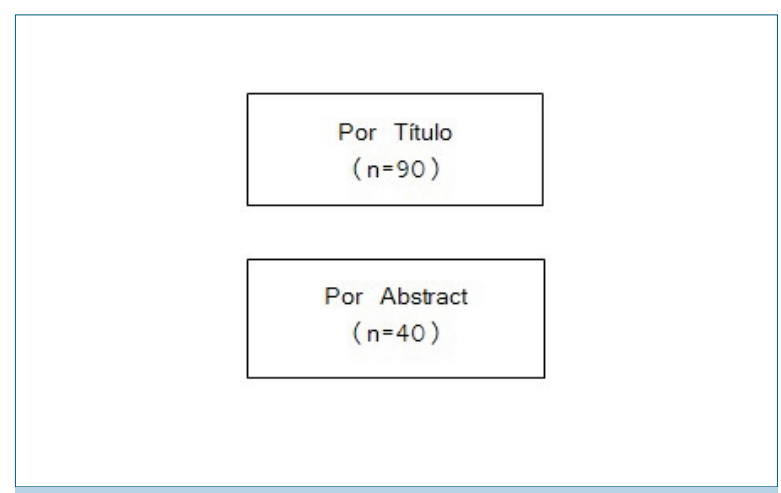

\section{Figura 1. Artículos incluidos en nuestra revisión.}

realizar una lectura completa. De estos 40 artículos se excluyeron 26 de ellos, quedando como resultado 14 trabajos incluidos en la presente revisión.

\section{Resultados}

Se resumen en la tabla 2.

\section{Indicaciones del uso del fijador externo}

En relación a la inmovilización inicial luego de la luxación de la rodilla, están descritos varios métodos para la estabilización temporal de la articulación.
Entre ellos destacamos técnicas tanto invasivas como no invasivas ${ }^{(8,11-23)}$.

Dentro de las primeras se destacan los fijadores externos, principalmente el del tipo sistema de fijación tubular AO (joint-spanning external fixation) y el fijador externo articulado (hinged joint fixation). Como medidas no invasivas se utilizan las férulas tanto termoplásticas como de yeso.

Es importante destacar además que el uso del método dependerá de la disponibilidad en el centro asistencial y de la lesión inicial.

De la totalidad de artículos revisados, 14 de ellos mencionan la fijación externa como parte del manejo de la luxación traumática de rodilla. Las indicaciones son principalmente: lesión vascular ${ }^{(8,11,12,16-22)}$, luxación expuesta ${ }^{(8,11,12,16,20-22)}$, inestabilidad en la articulación $(8,11,14-16,22,23$,$) , luxofracturas { }^{(8,13,14)}$, lesión en el aparato extensor $^{(14,21)}$.

Con respecto a la lesión vascular, los autores que defienden su uso, independientemente de si se realiza reparación vascular o no, sostienen que previene el trauma vascular adicional, y en el caso de que si requiera intervención, ofrecería mayor estabilidad y protección a la reparación ${ }^{(8,11,12,16-22)}$.

En el caso de las luxofracturas expuestas, éstas necesitan debridamiento quirúrgico y tienen alto riesgo de 


\begin{tabular}{|c|c|}
\hline Tabla 2 & \\
\hline $\begin{array}{l}\text { Artículo } \\
\text { Autores }\end{array}$ & $\begin{array}{l}\text { Tutor externo } \\
\text { Indicaciones }\end{array}$ \\
\hline $\begin{array}{l}\text { Conducta inicial en la luxación aguda de rodilla. Nuestra experiencia de } 20 \text { años. } \\
\text { - Dr. Facundo Gigante et al }\end{array}$ & $\begin{array}{l}\text { - Rodilla inestable } \\
\text { - Luxo fracturas } \\
\text { - Lesión vascular } \\
\text { - Lesiones expuestas }\end{array}$ \\
\hline $\begin{array}{l}\text { Luxación de rodilla. Tratamiento quirúrgico en casos agudos. } \\
\text { - Dr. Adolfo de los Ríos Giraldo }\end{array}$ & $\begin{array}{l}\text { - Rodilla inestable } \\
\text { - Cirugía vascular } \\
\text { - Lesión expuesta inestable. }\end{array}$ \\
\hline $\begin{array}{l}\text { Luxación expuesta de rodilla: ¿Qué hemos aprendido en los últimos } 20 \text { años? } \\
\text { - G. Xabier Carredano et al }\end{array}$ & $\begin{array}{l}\text { - Luxación expuesta } \\
\text { - Lesión vascular }\end{array}$ \\
\hline $\begin{array}{l}\text { Knee dislocation: Descriptive study of injuries } \\
\text { - Fabiano Kupczik et al }\end{array}$ & $\begin{array}{l}\text { - Luxación traumática } \\
\text { - Luxo fracturas }\end{array}$ \\
\hline $\begin{array}{l}\text { Fracture Dislocation of the Knee } \\
\text { - James Stannard, md }\end{array}$ & $\begin{array}{l}\text { - Rodilla inestable o que no se reduce } \\
\text { - Fractura de platillo tibial que provoca } \\
\text { acortamiento }\end{array}$ \\
\hline $\begin{array}{l}\text { Clinical and functional results of } 119 \text { patients with knee dislocations } \\
\text { - Nicholas R. Scarcella et al }\end{array}$ & - Rodilla inestable \\
\hline $\begin{array}{l}\text { Stage protocol for initial management of the dislocated knee } \\
\text { - Bruce A. Levy et al }\end{array}$ & $\begin{array}{l}\text { - Lesión vascular } \\
\text { - Inestabilidad } \\
\text { - Luxación expuesta } \\
\text { - Falla en la reducción } \\
\text { - Intolerancia a un brace }\end{array}$ \\
\hline $\begin{array}{l}\text { Knee dislocations and popliteal artery injury: A single centre experience from karachi } \\
\text { - Mehroze Zamir et al }\end{array}$ & - Reparación vascular \\
\hline $\begin{array}{l}\text { Demographics and injuries associated with knee dislocation } \\
\text { - Gilbert Moatshe et al }\end{array}$ & - Lesión vascular \\
\hline $\begin{array}{l}\text { Lesiones vasculares asociadas a luxación de rodilla: protocolo diagnóstico } \\
\text { - A. López- Hualda et al }\end{array}$ & $\begin{array}{l}\text { - Reparación vascular } \\
\text { - Reducción abierta }\end{array}$ \\
\hline $\begin{array}{l}\text { Dislocation and bicruciate lesions of the knee: Epidemiology and acute stage assessment in a } \\
\text { prospective series } \\
\text { - S. Lustig et al }\end{array}$ & $\begin{array}{l}\text { - Lesión vascular } \\
\text { - Luxación expuesta }\end{array}$ \\
\hline $\begin{array}{l}\text { Surgical management of posterior knee dislocation associated with extensor } \\
\text { apparatus rupture } \\
\text { - Chang- Cheng Liu et al }\end{array}$ & $\begin{array}{l}\text { - Lesión vascular } \\
\text { - Luxación expuesta } \\
\text { - Lesión del aparato extensor }\end{array}$ \\
\hline $\begin{array}{l}\text { Luxaciones de rodilla: Revisión de } 11 \text { casos } \\
\text { - B. Novoa Sierra et al }\end{array}$ & $\begin{array}{l}\text { - Luxaciones abiertas } \\
\text { - Lesión vascular con reparación } \\
\text { - Luxación incoercible }\end{array}$ \\
\hline $\begin{array}{l}\text { Importance of Early Diagnosis and Care in Knee Dislocations Associated with Vascular Injuries } \\
\text { - Victoria Teissier et al }\end{array}$ & - Rodilla inestable \\
\hline
\end{tabular}

complicaciones infecciosas. El fijador externo en estos casos favorece la rehabilitación del tejido dañado y permite además la movilidad del paciente ${ }^{(8,11,12,14,16)}$.

En el caso de las fracturas expuestas, la mayoría de los autores coinciden en el uso de fijación exter$\mathrm{na}^{(8,11,12,16,20-22)}$. Stannard y colaboradores ${ }^{(14)}$ propusieron utilizar fijadores externos en el caso de que la fractura no se mantuviera estable luego de la reducción o lesión del platillo tibial. La posición de reducción de la rodilla debería de ser de 20 grados de flexión ${ }^{(14)}$.
Los autores Scarcella ${ }^{(15)}$, López Hualda ${ }^{(19)}$, Novoa Sierra $^{(22)}$ y Victoria Teissier ${ }^{(23)}$, mencionan el manejo conservador con férulas para el caso de las lesiones que se mantuvieran estables o en aquellas en las que no hubiera acortamiento del miembro.

En relación al posicionamiento del fijador externo en el miembro lesionado, Levy ${ }^{(16)}$ propone la colocación de pines compatibles con la resonancia magnética y la colocación de dos de ellos en posición anterolateral en el fémur, a $5 \mathrm{~cm}$ proximales a la rótula, y a $2 \mathrm{~cm}$ distales an- 
teromediales en la tibia, distal al sitio de reconstrucción a futuro de los túneles para el ligamento cruzado anterior y posterior. Se debe tener precaución de no traccionar innecesariamente la articulación ${ }^{(8,15,19,23)}$.

No hay un consenso claro con respecto al tiempo en que deben dejarse los FFEE. Levy ${ }^{(16)}$, por ejemplo, lo mantiene durante tres a cuatro semanas, luego de lo cual lo retira y le coloca al paciente una férula externa articulada. De los Ríos Giraldo ${ }^{(11)}$ los deja por tiempo promedio de 6 semanas. mientras que Scarcella ${ }^{(15)}$ los deja como tiempo medio 6 a 8 semanas, Zamir ${ }^{(17)}$ los mantiene durante 6 semanas.

Queremos destacar que en la gran mayoría de los artículos no se mencionan protocolos sobre el tiempo de uso del fijador externo.

Dentro de las desventajas de su uso queremos destacar que es un método invasivo, aumenta el riesgo de artrofibrosis y la infección del trayecto de los pi$\operatorname{nes}^{(1,8,9,11,12,16,20-22,26)}$.

\section{Discusión}

Este trabajo tuvo como objetivo principal revisar la evidencia disponible hasta el momento, con un criterio temporal de veinte años, acerca de las indicaciones del uso del FFEE en la luxación traumática aguda de rodilla en la urgencia.

Desde el punto de vista metodológico revistió complejidad dada la heterogeneidad de artículos revisados y el enfoque dado por cada uno de los autores. Asimismo, existen numerosas publicaciones donde se hace hincapié en la reconstrucción de la lesión multiligamentaria.

En todos los artículos incluidos en nuestro trabajo se menciona la fijación externa como medida de estabilización inicial, con la premisa de convertir una inestabilidad multidireccional en una anteroposterior, así como también proteger, monitorizar y favorecer la recuperación de los tejidos blandos, brindar estabilidad a las reparaciones vasculares y a las reparaciones óseas agudas.

Esto coincide con las indicaciones propuestas por otros autores como Maslaris y Seroyer ${ }^{(1,10)}$, haciendo especial énfasis en la protección de la intervención vascular en caso de que esta sea necesaria. En cualquier caso, todos los autores coinciden en que la premisa fundamental es la reducción inmediata de la luxación para restituir las relaciones articulares normales y evitar daños secundarios adicionales, sobre todo a nivel vascular.

Si bien existe consenso a nivel mundial en cuanto a la indicación del uso de la fijación externa en el contexto de una lesión vascular, queremos destacar la revisión sistemática que realizaron los autores Ramírez-Bermejo, Gelber y Pujol ${ }^{(26)}$, siendo uno de sus objetivos valorar esta indicación y que se contrapone a lo anteriormente mencionado. Refieren que no pueden dar una justificación para su uso y que faltan estudios con series más grandes para respaldar esta decisión.

\section{Limitantes}

Queremos destacar que esta revisión incluye artículos de baja evidencia científica (nivel IV). Corresponden en su mayoría a series de casos, con diferentes matices en cuanto al manejo de la patología. Muchas de las indicaciones surgen de la experiencia personal de autores.

\section{Conclusión}

Hemos revisado la bibliografía actual acerca de las indicaciones del uso del fijador externo en la luxación traumática aguda de la rodilla.

Con respecto a la indicación de fijación externa, si bien es controvertido hasta el día de la fecha, parecería haber una clara tendencia a indicar su uso en los siguientes escenarios clínicos: luxación expuesta, politraumatizados, luxación incoercible, luxofracturas (fémur, tibia, platillo tibial), luxaciones asociadas a lesiones del aparato extensor, lesión vascular, síndrome compartimental, pacientes obesos e intolerancia a la férula u ortesis como método de inmovilización. Las ventajas de su uso son proveer de estabilidad a la articulación, la prevención de daño neurovascular y el control de las lesiones de partes blandas.

\section{Abstract}

Introduction: traumatic dislocation of the knee constitutes an unusual lesion in orthopedic pathology, the diagnosis of which is often missed. This is because it occurs in the context of multiple trauma patients, and failure to diagnose it may result in a potentially fatal outcome for the life-threatening compromise of the injured limb.

Objective: to perform a literature review of literature on indications for the use of the external fixator in the acute knee dislocation at the emergency room that has been available in the last twenty years.

Method: we conducted a systematized search by means of electronic search engines Cochrane, Lilacs, Scielo, Pubmed, Science direct and the Timbó portal. The search included 6495 articles and according to the criteria of inclusion and exclusion 14 studies were selected.

Results: the review highlights that most articles found are level of evidence IV. The use of the external fixator in the emergency room as part of the temporary stabilization and initial handling of the condition would be indicated in the following clinical scenarios: multiple trauma patients, vascular lesion, exposed dislocation, joint instability, recurrent dislocation, fracture disloca- 
tion, lesions of the extensor apparatus, morbid obesity, splint or orthosis intolerance.

Conclusion: systematization and the observation of protocols when it comes to the making of decisions enables the decrease of most complications associated to trauma pathologies. Actions must be geared to stabilizing patients first and to stabilize the knee joint. The external fixator is indicated for specific clinical scenarios. However, some of these indications are still a matter of debate.

\section{Resumo}

Introdução: a luxação traumática do joelho é uma lesão rara na patologia ortopédica. Em muitas ocasiões, seu diagnóstico passa despercebido, por ocorrer no contexto de pacientes politraumatizados, e que sua falha pode levar a um prognóstico potencialmente letal para o envolvimento vital do membro lesado.

Objetivo: realizar uma revisão da literatura disponível nos últimos vinte anos sobre as indicações do uso do fixador externo na luxação traumática aguda do joelho em pronto-socorro.

Materiais e métodos: foi realizada uma busca sistemática nas bases LILACS, SciELO, PubMed, Science Direct, na Biblioteca Cochrane e no portal Timbó. Foram obtidas 6.495 referencias dos quais 14 artigos foram selecionados de acordo com os critérios de inclusão e exclusão.

Resultados: a maioria dos artigos encontrados apresentam evidência de nível IV. O uso de fixação externa na emergência como parte da estabilização temporária e manejo inicial, seria indicado nos seguintes cenários clínicos: pacientes politraumatizados, lesão vascular, luxação exposta, instabilidade articular, luxação recorrente, luxação, lesões ao nível do aparelho extensor, obesidade mórbida, intolerância ao uso de tala ou órtese.

Conclusão: a sistematização e protocolização no momento da tomada de decisão é o que possibilita reduzir, em sua maioria, as complicações relacionadas à patologia traumática. Estas devem ter como objetivo em primeiro lugar estabilizar o paciente e, em segundo lugar, estabilizar a articulação do joelho. A fixação externa tem indicações em contextos clínicos específicos; no entanto, algumas delas ainda geram controvérsia.

\section{Bibliografía}

1. Maslaris A, Brinkmann O, Bungartz M, Krettek C, Jagodzinski M, Liodakis E. Management of knee dislocation prior to ligament reconstruction: what is the current evidence? Update of a universal treatment algorithm. Eur J Orthop Surg Traumatol 2018; 28(6):1001-15. doi: 10.1007/ s00590-018-2148-4.
2. Moatshe G, Chahla J, LaPrade RF, Engebretsen L. Diagnosis and treatment of multiligament knee injury: state of the art. J ISAKOS 2017; 2(3):152-61. doi: 10.1136/jisakos-2016-000072.

3. Court-Brown CM, Heckman JD, McQueen MM, Ricci WM, Tornetta III P, eds. Rockwood and green's fractures in adults. 8th ed. Philadelphia: Wolters Kluwer Health, 2015.

4. Cooper A. A treatise on dislocations and on fractures of the joints: fractures of the neck of the thigh-bone. 1923. Clin Orthop Relat Res 2007; 458:6-7. doi: 10.1097/BLO. 0b013e31803dee7c

5. A treatise on dislocations and on fractures of the joints. Edinb Med Surg J 1824; 21(79):370-97.

6. Birkett J. Compound dislocation of the knee. Lancet 1850; 2:703.

7. Plancher KD, Siliski JM. Dislocation of the knee. In: Plancher KD, Siliski JM, eds. Traumatic disorders of the knee. New York: Springer, 1994:315-31. doi: 10.1007/978-14612-4310-6 18 .

8. Gigante F, Miguel Lapera M, Pérez Zabala M, Pesolani M, Serpone A. Conducta inicial en la luxación aguda de rodi1la. Nuestra experiencia de 20 años. Artroscopía 2016; 23(2):61-9.

9. McKee L, Ibrahim MS, Lawrence T, Pengas IP, Khan WS. Current concepts in acute knee dislocation: the missed diagnosis? Open Orthop J 2014; 8:162-7. doi: 10.2174/1874325001408010162.

10. Seroyer ST, Musahl V, Harner CD. Management of the acute knee dislocation: the Pittsburgh experience. Injury 2008; 39(7):710-8. doi: 10.1016/j.injury.2007.11.022.

11. De Los Ríos A. Luxación de rodilla: tratamiento quirúrgico en casos agudos. Rev Colom Ortop Traumatol 2005; 19(3):34-43.

12. Carredano GX, Valderrama R J, Montenegro BD, Lizama CP, Bernal BN, Valderrama SI, et al. Luxación expuesta de rodilla: ¿Qué hemos aprendido en los últimos 20 años? Rev Chil Ortop Traumatol 2018: 59(2):47-54.

13. Kupczik F, Schiavon MEG, Vieira LA, Tenius DP, Fávaro RC. Knee dislocation: descriptive study of injuries. Rev Bras Ortop 2013; 48(2):145-51. doi: 10.1016/j.rboe. 2012.10.002.

14. Stannard J. Fracture dislocation of the knee. J Knee Surg 2016; 29(4):300-2. doi: 10.1055/s-0036-1583177.

15. Scarcella NR, Weinberg DS, Bowen S, Vallier HA. Clinical and functional results of 119 patients with knee dislocations. J Orthop Trauma 2017; 31(7):380-6. doi: 10.1097/ BOT.0000000000000839.

16. Levy BA, Krych AJ, Shah JP, Morgan JA, Stuart MJ. Staged protocol for initial management of the dislocated knee. Knee Surg Sports Traumatol Arthrosc 2010; 18(12):1630-7. doi: 10.1007/s00167-010-1209-y.

17. Zamir M, Noor SS, Rahim Najjad MK, Aliuddin AM, Ghilzai AK. Knee dislocations and popliteal artery injury: a single centre experience from Karachi. J Pak Med Assoc. 2014; 64(12 Suppl 2):S91-4. 
18. Moatshe G, Dornan GJ, Løken S, Ludvigsen TC, LaPrade RF, Engebretsen L. Demographics and injuries associated with knee dislocation: a prospective review of 303 patients. Orthop J Sports Med 2017; 5(5):2325967117706521. doi: $10.1177 / 2325967117706521$.

19. López-Hualda A, Valencia-García H, Martínez-Martín J. Lesiones vasculares asociadas a luxación de rodilla: protocolo diagnóstico. Rev Esp Cir Ortop Traumatol 2012; 56(4):260-6. doi: 10.1016/j.recot.2011.12.005.

20. Lustig S, Leray E, Boisrenoult $\mathbf{P}$, Trojani C, Laffargue $\mathbf{P}$, Saragaglia D, et al. Dislocation and bicruciate lesions of the knee: epidemiology and acute stage assessment in a prospective series. Orthop Traumatol Surg Res 2009; 95(8):614-20. doi: 10.1016/j.otsr.2009.10.005.

21. Liu CC, Gao X, Xu M, Knog ZG. Surgical management of posterior knee dislocation associated with extensor apparatus rupture. Knee 2017; 24(5):940-8. doi: 10.1016/ j.knee. 2017.05.023.

22. Novoa Sierra B, Estrems Díaz V, Ribas García-Peñuela JS, Bru Pomer A. Luxaciones de rodilla: revisión de 11 ca- sos. Rev Esp Cir Ost 2015; 50(262):123-7. Disponible en: http://www.cirugia-osteoarticular.org/adaptingsystem/intercambio/revistas/articulos/2393_123.pdf. (Consulta: 16 febrero 2020).

23. Teissier V, Tresson P, Gaudric J, Davaine JM, Scemama C, Raux M, et al. Importance of early diagnosis and care in knee dislocations associated with vascular injuries. Ann Vascular Surg 2019; 61:238-45. doi: 10.1016/j.avsg.2019. 04.016.

24. Niall D, Nutton RW, Keating JF. Palsy of the common peroneal nerve after traumatic dislocation of the knee. J Bone Joint Surg Br 2005; 87(5):664-7.

25. McKee L, Ibrahim MS, Lawrence T, Pengas IP, Khan WS. Current concepts in acute knee dislocation: the missed diagnosis? Open Orthop J 2014; 8:162-7. doi: 10.2174/1874325001408010162. eCollection 2014.

26. Ramírez-Bermejo E, Gelber PE, Pujol N. Management of acute knee dislocation with vascular injury: the use of the external fixator. A systematic review. Arch Orthop Trauma Surg 2020. doi: 10.1007/s00402-020-03684-0.

Contribución de autores

Mateo Pamparato, ORCID 0000-0002-2156-7300. Revisión sistemática, discusión y conclusiones

Juan M. Del Castillo, ORCID 0000-0002-3239-4337. Revisión sistemática, discusión y conclusiones.

Luis Francescoli, ORCID 0000-0002-7048-7630. Discusión y conclusiones. 